\title{
IPN Membranes for the Pervaporation of Ethanol/Water Mixture
}

\author{
Y. K. Lee, I. S. Sohn, E. J. Jeon, and S. C. Kim \\ Department of Chemical Engineering, \\ Korea Advanced Institute of Science and Technology, \\ P.O. Box 150, Chongyangni, Seoul, 130-650, Korea
}

(Received November 26, 1990)

\begin{abstract}
Hydrophilic/hydrophobic, cationic/anionic hetero geneous IPN membranes as well as hydrophilic/hydrophilic homogeneous IPN membranes were prepared and the pervaporation characteristics of the membranes for the separation of ethanol/water mixture were elucidated. The computer simulation of the continuous rectangular pervaporator was conducted and the membrane characteristics for the optimum performance was analyzed. The IPN membrane characteristics were estimated based on the heterogeneous and homogeneous model and the predicted properties were compared with experimental data.

KEY WORDS IPN / Membrane / Pervaporation / Continuous Pervaporator / Hydrophilic-hydrophobic / Hydrophilic-hydrophilic / cationic-anionic /
\end{abstract}

Interpenetrating Polymer Network (IPN) Membranes can be considered as a potential micro-phase separated membrane materials since they provide amphoteric properties with controlled morphology. IPN is defined as the mixture of two or more crosslinked polymers with partial or total physical interlocking present among the component polymers. The IPN material is viewed as the polymer blend of the crosslinked polymers, thus the advantage of combining two insoluble thermosetting polymers as the components should be utilized. This advantage is particularly important for the membranes with liquid separation since the transporting phase should not be dissolved in the liquid media while still acting as the selective transporting phase and the separation selectivity is enhanced with the presence of the neighboring non-transporting phase which reduces the swelling of the transporting phase thus restricting the so-called "plasticization effect". The morphology of the IPN can be controlled to give membrane materials with different domain size or with different degree of phase separation by varying the catalyst and crosslinking agent concentration and also by varying the reaction pressure and temperature. The early stoppage of the phase separation during the spinodal decomposition with the formation of interlocked network provides membranes of co-continuous morphology, and this is useful for the amphoteric IPN membranes of hydrophylic/hydrophobic, cationic/anionic, polar/nonpolar and glassy/rubbery IPN membranes.

The presence of physical interlocking in homogeneous IPN's where there is no phase separation, provides additional physical crosslinking due to interpenetration and gives membrane materials with enhanced mechanical strength and reduced swelling.

\section{EXPERIMENTAL}

Hydrophilic polyurethane(PU) was based on the reaction product of 
hexamethylene diisocyanate(HDI), polyethylene glycol(PEG) of 1000 mol.wt. and trimethylol propane(TMP) as the crosslinking agent. The hydrophilic/hydrophobic IPN membrane was prepared by mixing polyurethae oligomer, TMP, styrene monomer(SM), divinyl benzene(DVB) and benzoyl peroxide(BPO) and simultaneously polymerizing PU network (by condensation mechanism) and PS network (by free radical mechanism) in thin film form. Synthetic procedures were reported elsewhere ${ }^{1,2}$. The cationic PU network was synthesized by employing $\mathrm{N}-$-methyl diethanolamine(MDEA) as a chain extender and the tertiary amine group in the network was later quarternized by benzyl chloride. The anionic poly(methyl methacrylate-co-acrylic acid) (P(MMA-AA)) network was synthesized by reacting MMA, AA and ethylene glycol dimethacrylate (EGDMA, as the crosslinking agent) simultaneously with the PU component and later neutralized by $\mathrm{NaOH}^{3,4}$

The homogeneous IPN membranes of hydrophobic poly(styrene-coacrylonitrile) (PSAN) and hydrophilic polyacrylic acid (PAA) was also synthesized by sequential polymerization. The PSAN membranes were swollen in AA, EGDMA monomer mixture and polymerized in the second stage ${ }^{5}$. The homogeneous IPN membranes of hydrophilic PU and hydrophilic PHEMA were prepared also by sequential polymerization. The hydrophilic PU was swollen in HEMA, EGDMA mixture and subsequently polymerized ${ }^{7}$. The pervaporation experiments were conducted as described before and the swelling ratio, separation factor and the permeability of water in the water-ethanol mixture were measured.

\section{RESULTS AND DISCUSSION}

In the hydrophilic PU and hydrophobic PS IPN membranes, the effect of the synthesis pressure on the pervaporation characteristics was evaluated . the separation factor of water increased due to the formation of the co-continuous morphology and the reduction of domain size (Figure 1) when the IPN membranes was synthesized under high pressure. The co-continuous morphology of the hydrophilic and hydrophobic phase will result in the reduced swelling of the membrane in aqueous solution due to the restriction of swelling of the hydrophilic PU by the neighboring hydrophobic PS, and the plasticizing effect in the membrane is reduced, thus increasing the separation factor. Furthermore, the wet strength of the membrane was enhanced about eight times in the IPN membrane by the incorporation of the hydrophobic PS component.

In the cationic PU and anionic $\mathrm{P}(\mathrm{MMA}-\mathrm{AA})$ membranes the effect of the composition and the ionic concentration on the pervaporation characteristics were analyzed. The ion concentration affected the degree of swelling and the affinity toward water. The separation factor showed maximum value when the ion concentration in the membrane was around $1500 \overline{\mathrm{Mi}}$ (the average moleculer weight between ionic centers). A synergistic effect was also observed in the separation factor of the membrane when the PU composition was varied. The separation factor reached maximum value at the PU/P(MMA-AA) $10 / 90$ IPN by the restriction in swelling due to the increase in physical interlocking between the component polymers in the IPN membranes.

The computer simulation of the actual continuous pervaporation process through rectangular channels of $3 \times 0.3 \times 100 \mathrm{~cm}^{3}(\mathrm{WxHxL})$ (Figure 2) shows that the concentration polarization across the height of the channel was not significantly affected by varying the separation factor of the membrane but significant effects were observed by varying the permeability ${ }^{7}$. It was observed that the performance of the continuous pervaporation process which was quantified by the objective function of $\mathrm{QxC}$ (where $\mathrm{Q}$ is the flow rate of the retentate (ethanol production rate) and $\mathrm{C}$ is the ethanol concentration in the retentate) was high when the membrane had higher permeability in the initial section of the channel when the feed ethanol concentration was low, but in the later section of the channel when the feed ethanol concentration was high, membranes with higher separation factor gave better performance. The 


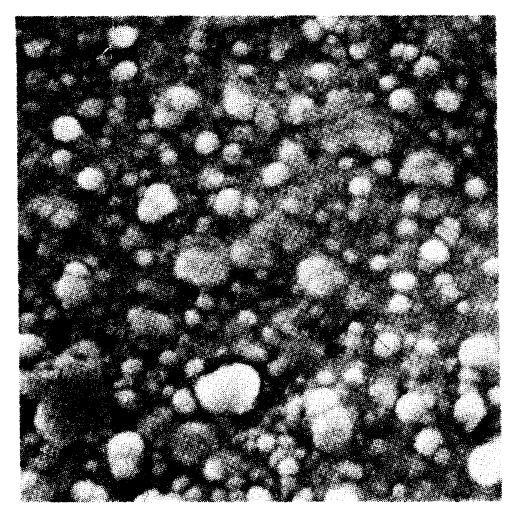

(a)

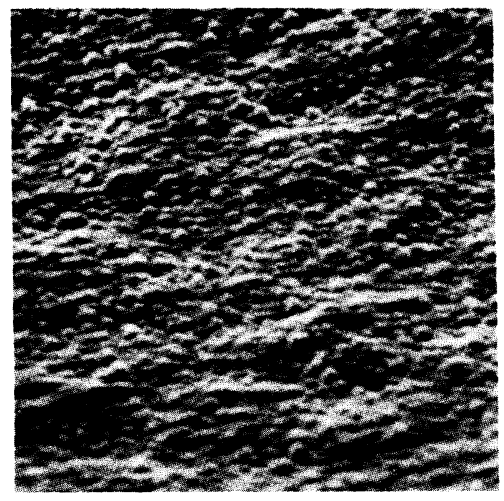

(c)

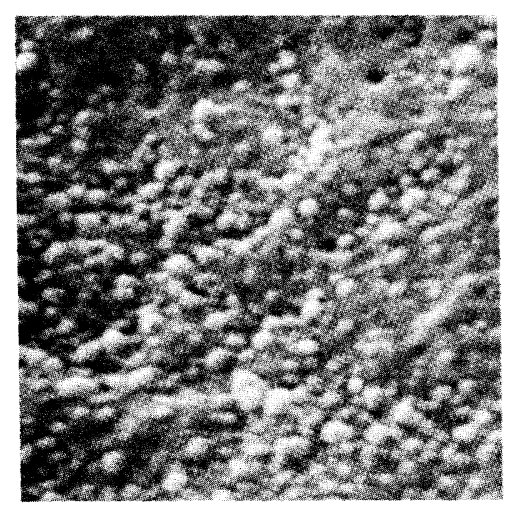

(b)

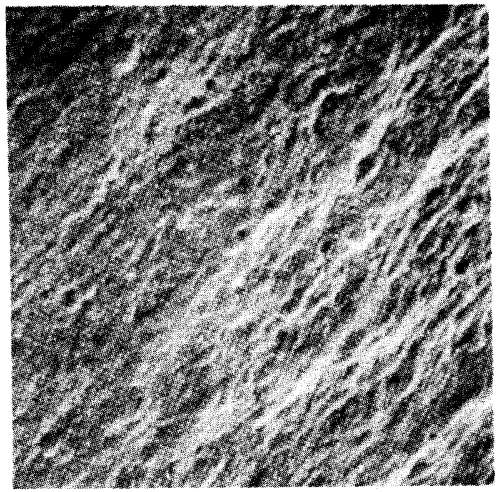

(d)

Figure 1. Scanning electron micrographs of the IPN membranes of PU/PS 50/50 synthesized with varying pressure. a) atmospheric b) $100 \mathrm{MPa} \mathrm{c}$ ) $250 \mathrm{MPa}$ d) $500 \mathrm{MPa}$

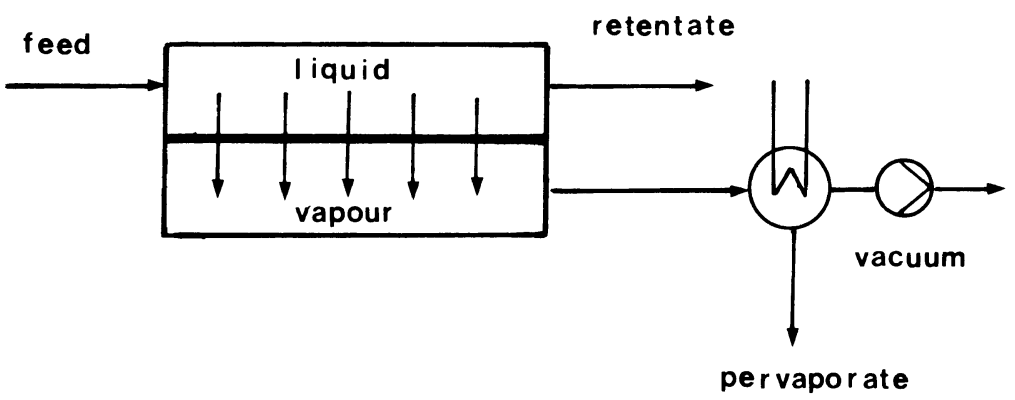

Figure 2. Continuous pervaporation process through rectangular channel. 
employment of homogeneous IPN's of hydrophobic PSAN/PAA with different PAA content gave optimum performance in the continuous channel pervaporator. The three stage pevaporator with PSAN/PAA 40/60 IPN membranes (high permeability with low separation factor) in the first stage and with PSAN/PAA 60/40 IPN membrane (high separation factor with low permeability) in the second and third stage gave production of ethanol of $94 \%$ in the retentate (Figure 3 ) in the $3 \mathrm{~cm} \mathrm{~W} \mathrm{x}$ $0.3 \mathrm{~cm} \mathrm{H} \times 100 \mathrm{~cm} \mathrm{~L}$ channel pervaporator with feed concentration of $10 \%$ ethanol and flow rate of $100 \mathrm{~g} / \mathrm{hr}$.

The prediction scheme of pervaporation characteristics of heterogeneous IPN membranes of cationic PU and anionic P(MMA-AA) ${ }^{4}$ and of homogeneous IPN membranes of cationic PU and hydrophilic PHEMA using heterogeneous and homogeneous mixture models were devised ${ }^{8}$. The single component membrane characteristics of cationic PU, anionic P(MMA-AA) and hydrophilic PHEMA were determined using the following "six-coefficients model" of Brun.

$$
\begin{aligned}
& D_{i}=D_{i o e x p} \\
& D_{j}=D_{j o e x p}
\end{aligned}\left(\begin{array}{l}
A_{i i} C_{i}+A_{i j} C_{j} \\
A_{j i} C_{i}+A_{j j} C_{j}
\end{array}\right\}
$$

Table 1 lists the six coefficients determined from the computer regression of the actual experimental data of separation factor and permeability in different feed

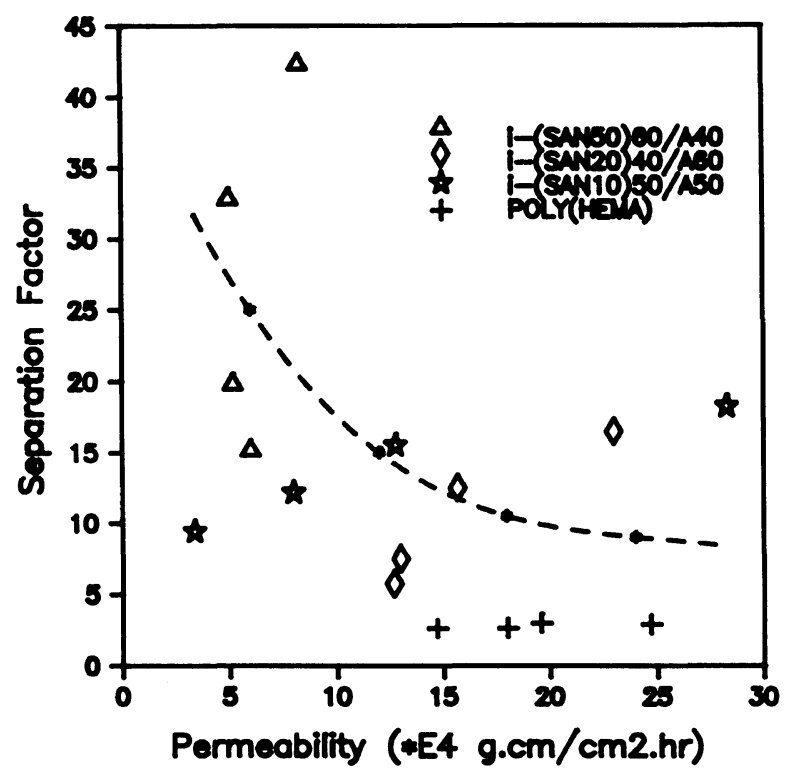

Figure 3. Characteristics of four membranes at various feed composition.

Table 1. Computed coefficients of the model for the pervaporation of water/ethanol through cationic PU, anionic P(MMA-AA), PU and PHEMA membranes

\begin{tabular}{lllll}
\hline & $\begin{array}{l}\text { cationic } \\
\text { PU }\end{array}$ & $\begin{array}{l}\text { anionic } \\
\text { P(MMA-AA) }\end{array}$ & PU & PHEMA \\
\hline $\mathrm{D}_{\mathrm{io}}\left(\mathrm{cm}^{2} / \mathrm{sec}\right)$ & $1.767 \times 10^{-7}$ & $1.681 \times 10^{-7}$ & $1.264 \times 10^{-7}$ & $1.203 \times 10^{-7}$ \\
$\mathrm{D}_{\mathrm{jo}}\left(\mathrm{cm}^{2} / \mathrm{sec}\right)$ & $1.969 \times 10^{-7}$ & $0.508 \times 10^{-7}$ & $0.847 \times 10^{-7}$ & $0.503 \times 10^{-7}$ \\
$\mathrm{~A}_{\mathrm{ii}}\left(\mathrm{cm}^{3} / \mathrm{g}\right)$ & 1.0242 & 4.9749 & 10.11 & 4.32 \\
$\mathrm{~A}_{\mathrm{ij}}\left(\mathrm{cm}^{3} / \mathrm{g}\right)$ & 9.4527 & 4.3611 & 6.91 & 7.21 \\
$\mathrm{~A}_{\mathrm{ji}}\left(\mathrm{cm}^{3} / \mathrm{g}\right)$ & 0.9854 & 3.4358 & 8.18 & 5.13 \\
$\mathrm{~A}_{\mathrm{jj}}\left(\mathrm{cm}^{3} / \mathrm{g}\right)$ & 4.4868 & 5.1495 & 7.44 & 3.48 \\
\hline
\end{tabular}


concentrations for the single component membrane materials. Figure 4 shows the predicted permeability and separation factor of the anionic P(MMA-AA) membrane from the six-coefficients model and the sorption data and the actual experimental data, which shows good agreement. Similar level of agreement betweem the computed characteristics and the experimental results was observed in cationic PU and PHEMA membranes.

The combination of these single component membranes in IPN form was conducted according to the morphology of the IPN membranes. In homogeneous PU/PHEMA membranes, both the logarithmic mixing rule and the arithmetic mixing rule was applied and the predicted IPN characteristics were quite close to the actual data (Figure 5). In the heterogeneous IPN membranes of cationic PU and anionic $\mathrm{P}(\mathrm{MMA}-\mathrm{AA})$, a typical island-sea dispersion was observed for the PU/P(MMA-AA) $10 / 90$ IPN and co-continuous morphology for the PU/P(MMA-AA) 30/70 IPN through electron microscopy. A heterogeneous model of island-sea type morphology was used and the predicted characteristics of the $\mathrm{PU} / \mathrm{P}(\mathrm{MMA}-\mathrm{AA}) 10 / 90 \mathrm{IPN}$ membrane were in quite good agreement with the actual data (Figure 6) The co-continuous model also gives close predictions for the

U/P(MMA-AA) 30/70 IPN membranes (Figure 7)

\section{ACKNOWLEDGMENT}

The authors wish to acknowledge support of the Ministry of Science and Technology of the Republic of Korea and the Korea Science and Engineering Foundation.

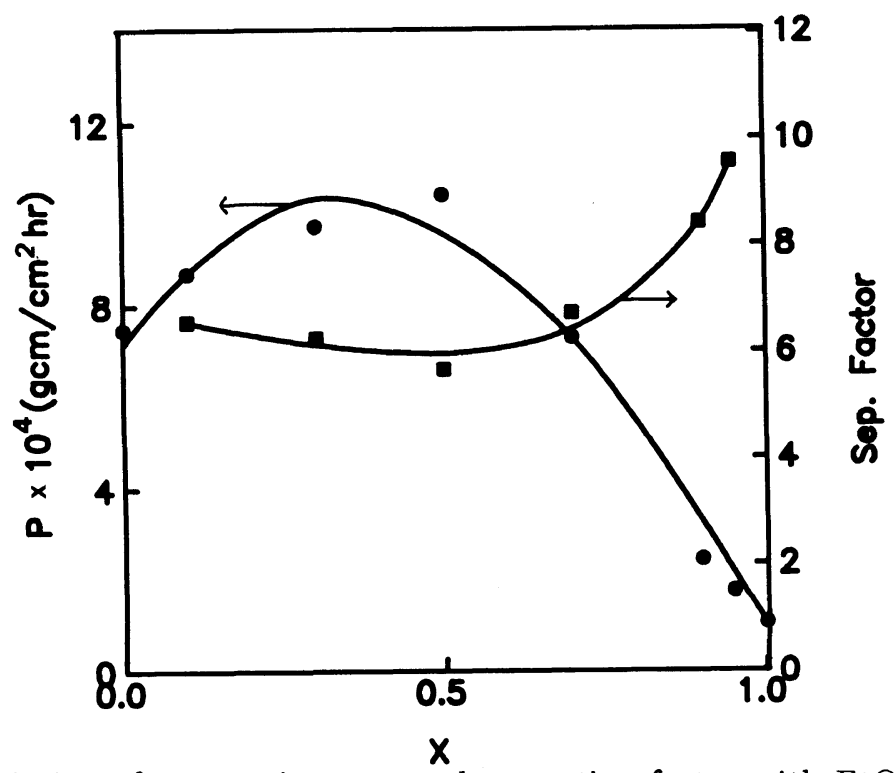

Figure 4. Variation of permeation rate and sepsration factor with EtOH wt.fraction in the pervaporation of water-EtOH mixtures through anionic $\mathrm{P}(\mathrm{MMA}-\mathrm{AA})$ membrane. The model(solid curves) is compared with the experimental data 


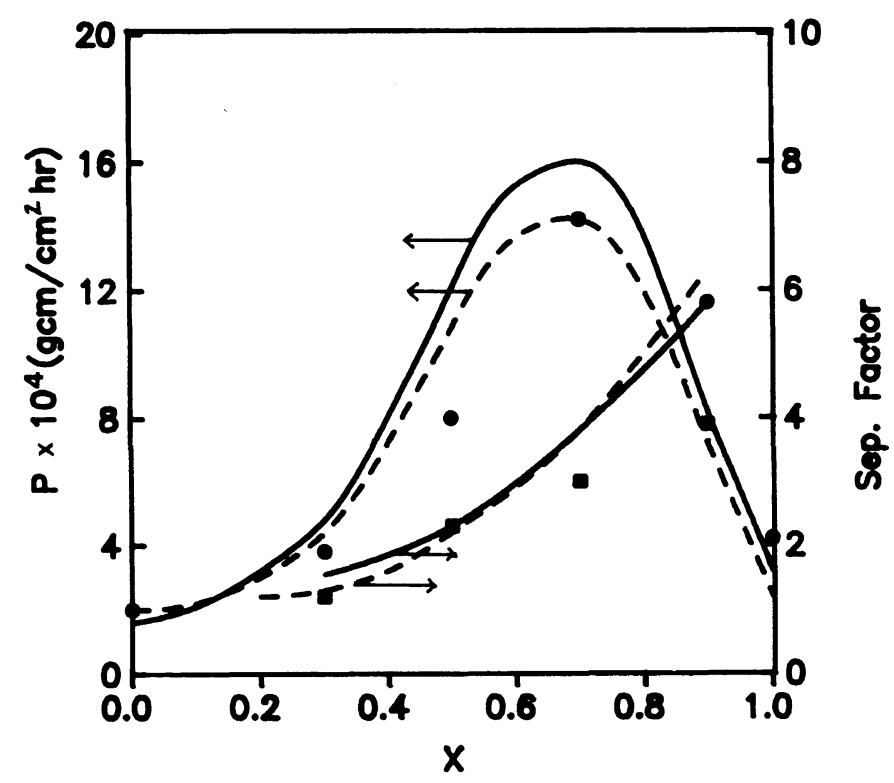

Figure 5. Variation of permeation rate and separation factor with EtOH wt.fraction in the pervaporation of water-EtOH mixtures through PU/PHEMA 40/60 membrane. The model is compared with the experimental data. (solid lines:arithmatic average values are used., dashed lines:logarithmic values are used.)

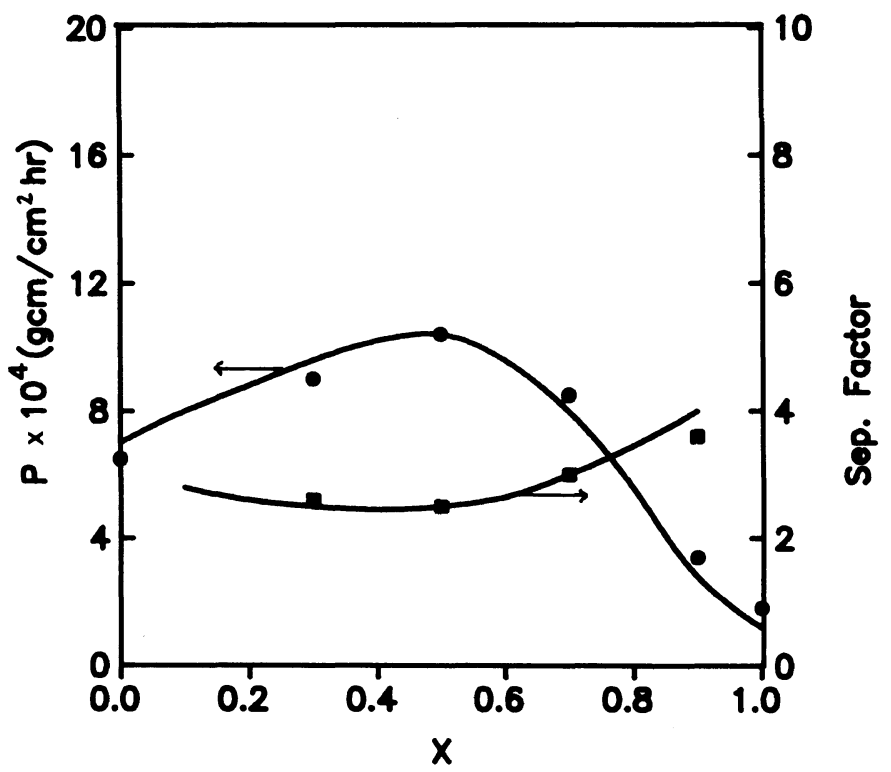

Figure 6. Variation of permeation rate and separation factor with EtOH wt.fraction in the pervaporation of water-EtOH mixtures through cationic/anionic $\mathrm{PU} / \mathrm{P}$ (MMA-AA) $10 / 90$ membrane. The model(solid curves) is compared with the experimental data. (P(MMA-AA) continuous) 


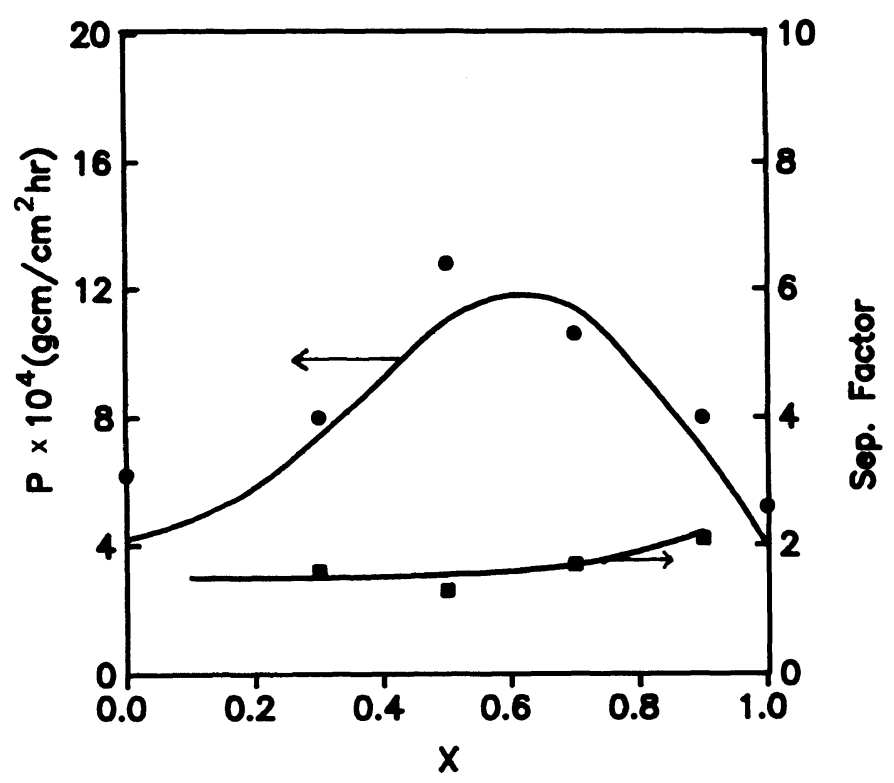

Figure 7. Variation of permeation rate and separation factor with EtOH wt.fraction in the pervaporation of water-EtOH mixtures through cationic/anionic $\mathrm{PU} / \mathrm{P}$ (MMA-AA) 30/70 membrane. The model(solid curves) is compared with the experimental data.(cocontinuous)

\section{REFERENCE}

1. J. H. LEE and S. C. Kim, Macromolecules, 19, 644(1986).

2. J. H. Lee and S. C. Kim, "Polymers for Advanced Technology", M. Lewin Ed., VCH Pub., New York (1988), p683.

3. Y. K. Lee and S. C. Kim, Polymer Bulletin, 20, 261(1988).

4. Y. K. Lee, T. M. Tak, D. S. Lee and S. C. Kim, J.Membrane Science, Elsevier, Amsterdam, 52, 157(1990).

5. H. J. Lee and S. C. Kim, MS Thesis, KAIST (1990).

6. S. H. Cho and S. C. Kim, MS Thesis, KAIST (1989).

7. I. S. Sohn and S. C. Kim, unpublished result.

8. E. J. Jeon and S. C. Kim, to be published. 